\title{
Equivalência semântica da versão brasileira da Social Avoidance and Distress Scale (SADS)
}

\author{
Semantic equivalence of the Brazilian Portuguese Version of the Social Avoidance \\ and Distress Scale (SADS)
}

\author{
Michelle Nigri Levitan'1, Isabella Nascimento², Rafael C. Freire ${ }^{3}$, Marco André Mezzasalma \\ Antonio Egidio Nardi ${ }^{5}$
}

\begin{abstract}
${ }^{1}$ Aluna, Estágio Probatório para Mestrado, Programa de Pós-Graduação, Instituto de Psiquiatria, Universidade Federal do Rio de Janeiro (UFRJ), Rio de Janeiro, RJ. ${ }^{2}$ Doutora em Psiquiatria. Instituto de Psiquiatria, UFRJ. Bolsista de Produtividade em Pesquisa, CNPq. Médica Psiquiatra, Instituto de psiquiatria, UFRJ. ${ }^{3}$ Mestrando, Programa de Pós-Graduação, Instituto de Psiquiatria, UFRJ. ${ }^{4}$ Mestre em Psiquiatria e aluno, Programa de PósGraduação do Instituto de Psiquiatria, UFRJ. Médico Psiquiatra, Instituto de Psiquiatria, UFRJ. ${ }^{5}$ Livre docente e professor associado, Instituto de Psiquiatria, UFRJ. Professor adjunto, Faculdade de Medicina, Instituto de Psiquiatria, UFRJ.
\end{abstract}

\section{Resumo}

Introdução: É crescente a produção científica brasileira na adaptação de instrumentos internacionais da fobia social. A adaptação transcultural é o primeiro passo na realização de comparações entre diferentes populações e se apresenta como um método que envolve pouco custo financeiro. O presente estudo consistiu no processo de equivalência semântica da Social Avoidance and Distress Scale para sua utilização na população brasileira de diferentes níveis socioeconômicos.

Métodos: O processo envolve duas traduções e retrotraduções realizadas por avaliadores independentes, avaliação das versões com elaboração de uma versão sínteses e pré-teste comentado.

Resultados: Para cada item do instrumento, apresentam-se os resultados das quatro etapas. A maioria dos participantes não apresentou dificuldades na compreensão do instrumento.

Conclusão: A utilização de duas versões de tradução e retrotradução, discussão sobre a versão síntese e a interlocução com a população-alvo proporciona maior segurança ao processo de equivalência semântica.

Descritores: Adaptação transcultural, escalas de ansiedade social, equivalência semântica e fobia social.

\begin{abstract}
Introduction: There has been a growing scientific production on the adaptation of international instruments for social phobia. The cross-cultural adaptation is the first stage on the comparisons between different populations and presents the advantage of a low financial cost. This paper consisted in the process of semantic equivalence of the Social Avoidance and Distress Scale for the Brazilian population of different sociocultural levels.

Methods: The semantic equivalence involved two translations and back-translations performed by two independent evaluators, an evaluation of the versions and the development of a synthetic version, and a commented pretest.

Results: The results of the four stages were showed for each item of the instrument. Most participants had no difficulties in understanding the instrument.

Conclusion: Use of two versions of translations, critical appraisal of the versions, and assessment of the target population provides more safety to the process of semantic equivalence.

Keywords: Cross-cultural adaptation, social anxiety scales, semantic equivalence, social anxiety.
\end{abstract}




\section{Introdução}

A fobia social (FS) caracteriza-se por uma experiência de medo intenso de ser humilhado ou julgado de forma negativa em situações sociais ${ }^{1}$. Em uma situação de medo, as pessoas apresentam sintomas somáticos, como rubor facial, palpitações e tremores. A ansiedade muitas vezes leva à evitação dos ambientes temidos, que variam de situações discretas a todos os contatos interpessoais ${ }^{2,3}$.

Instrumentos auto-aplicáveis para FS estão sendo culturalmente adaptados em muitos países ${ }^{4-7}$, apresentando algumas vantagens, como tempo e custo reduzidos na sua aplicação. Ao utilizar um instrumento já desenvolvido e sistematicamente usado em ensaios com FS, os pesquisadores podem facilmente comparar os resultados entre diferentes amostras ${ }^{8}$ e construir dados transculturais 9 .

A validação é o processo que examina até que ponto os resultados das medições correspondem ao estado real e/ou presença do fenômeno sendo mensurado ${ }^{10}$. Estudos de validação transcultural de escalas permitem que os pesquisadores aumentem o conhecimento psicométrico e façam comparações entre os dados encontrados em diferentes países ${ }^{11}$.

Aspectos transculturais podem modificar os resultados encontrados em amostras distintas, não porque sejam realmente diferentes, mas porque podem refletir características inerentes da amostra (não diretamente relacionadas à condição estudada). Ao adaptar escalas para diferentes idiomas, é importante assegurar que o sentido das questões e as instruções das escalas estejam sendo interpretados igualmente pelos dois idiomas ${ }^{12}$. O primeiro estágio da validação é a adaptação transcultural, que sistematicamente compara e analisa a versão da escala a ser utilizada em uma cultura específica.

Apesar da falta de consenso em relação à melhor metodologia indicada para conduzir uma equivalência semântica entre escalas, a abordagem recomendada inclui retrotradução realizada por dois tradutores independentes ${ }^{13,14}$. Neste modelo, o indivíduo bilíngüe traduz da língua-fonte para a língua-alvo, e outro tradutor "cego" traduz de volta para a língua-fonte. Após este processo, comparações são realizadas para identificar discrepâncias entre a fonte e o alvo, seguidas de discussões com os tradutores bilíngües originais ${ }^{15} \mathrm{e}$ da construção de uma versão sintética.

Embora existam algumas escalas que sirvam de instrumentos diagnósticos para FS, poucos instrumentos avaliam as limitações sociais e sentimentos negativos que o sujeito pode sentir em contextos sociais. A Social Avoidance And Distress Scale (SADS) ${ }^{16}$ consiste de 28 itens que avaliam dois aspectos da ansiedade social caracterizada por evitação de situações sociais e o desconforto sentido.
A escala consiste de 28 itens, e cada um deles é classificado como "verdadeiro" ou "falso". O respondente recebe um ponto para cada um dos itens classificados de "verdadeiro" (15 itens) e para cada um classificado como "falso" (13 itens). Pacientes socialmente fóbicos geralmente apresentam escores mais altos e espera-se que sintam desconforto e evitem situações sociais ${ }^{17}$. Na versão original, testada em universidades estadunidenses, a SADS teve excelente consistência interna de 0,94 e correlação significativa com as medições de sensibilidade do público, ansiedade e ansiedade social. Dados de confiabilidade teste-reteste foram obtidos de uma amostra de alunos por um período de 1 mês, com variação de 68 a $79^{17}$.

Parece que a SADS é mais adequada para avaliação de mal-estar geral e evitação do que de ansiedade social ${ }^{18}$, em razão do fato de que indivíduos com outros distúrbios de ansiedade também apresentam altos escores nesta escala. Assim, a SADS parece ser sensível para mal-estar emocional, mas não específica para FS, exigindo, portanto, cuidado em seu uso ${ }^{19}$. A SADS foi escolhida devido à simplicidade e boas propriedades psicométricas do instrumento. Uma vez que não há dados sobre adaptação cultural para o português brasileiro, decidimos desenvolver a tradução e adaptação cultural da versão em português brasileiro da SADS.

Este estudo integra o grande volume de produção científica brasileira sobre $\mathrm{FS}^{19-30}$. Alguns desses estudos ${ }^{19,24,25,29,30}$ concentraram-se na validação e adaptação transcultural de escalas, o que nos permite expandir os achados de estudos internacionais, uma vez que podemos replicar o estudo na população brasileira. Esses artigos apresentam notoriedade importante, já que reforçam a pesquisa sobre FS em centros especializados.

Este estudo tem por objetivo realizar uma equivalência semântica da versão em inglês da SADS para o português em uma amostra heterogênea da população brasileira.

\section{Métodos}

O processo de equivalência semântica adotado é parte da adaptação transcultural baseada no roteiro proposto por Herdman et al. ${ }^{31}$. Este processo, recentemente empregado no Brasil por Reichenheim et al. ${ }^{32}$, consiste de quatro etapas: tradução, retrotradução, avaliação de equivalência semântica e pré-teste na população-alvo com a elaboração de uma versão sintética.

Na primeira etapa, duas traduções do instrumento original em inglês foram realizadas por dois tradutores bilíngües (IN, MN), ambos fluentes na língua inglesa. $\mathrm{Na}$ segunda etapa, as duas traduções foram retrotraduzidas para o inglês, também de forma independente e cega à escala original, por dois tradutores 
bilíngües (RF, BN), que também eram fluentes em inglês. Três dos tradutores eram da área de psiquiatria, e um era falante fluente de inglês.

A terceira etapa consistiu da avaliação de equivalência semântica, realizada pelos dois autores (ML, AE), e da elaboração de uma versão sintética. A equivalência entre o instrumento original e cada uma das retrotraduções foi primeiramente avaliada e, a seguir, cada item do instrumento original foi comparado a seu correspondente na versão em português. Para compor a versão sintética, alguns itens foram incorporados de uma das duas versões, integralmente ou modificados pelo grupo, ao passo que outros itens se originaram da combinação de duas versões. O resultado desta combinação era por vezes modificado para melhor atender aos critérios de equivalência semântica.

Foi realizado um pré-teste com a versão sintética, aplicado a oito pessoas com escolaridade elementar ( 8 anos de estudo), oito com escolaridade intermediária
(11 anos de estudo) e oito com ensino superior (diploma universitário). Os indivíduos eram adultos selecionados entre os estudantes e funcionários de nossa universidade. Eles não apresentavam histórico de distúrbios mentais e foram submetidos a uma entrevista aberta pelos autores através de entrevista clínica estruturada para o DSM$\mathrm{IV}^{33}$ para eliminar qualquer possibilidade de diagnóstico. Todos os participantes comentaram sobre a versão sintética, apontando dificuldades e sugerindo palavras ou termos que pudessem ser entendidos com maior facilidade. Considerando essas sugestões, desenvolveuse uma versão final da SADS para o português brasileiro.

\section{Resultados}

A Tabela 1 mostra o instrumento original, as traduções (T1 e T2), suas respectivas retrotraduções (B1 e B2) e a versão sintética (antes das alterações pré-teste).

Tabela 1 - Instrumento original, traduções, retrotraduções e a versão sintética da Social Avoidance and Distress Scale

\begin{tabular}{|c|c|c|c|}
\hline Versão original & Traduções & Retrotraduções & Versão sintética \\
\hline \multirow[t]{2}{*}{$\begin{array}{l}\text { 1. I feel relaxed even } \\
\text { in unfamiliar social } \\
\text { situations }\end{array}$} & $\begin{array}{l}\text { T1. Eu me sinto à vontade em } \\
\text { situações sociais novas }\end{array}$ & $\begin{array}{l}\text { B1. I feel comfortable in new } \\
\text { social occasions }\end{array}$ & $\begin{array}{c}\text { Eu me sinto à vontade em } \\
\text { situações sociais que não tenho } \\
\text { familiaridade }\end{array}$ \\
\hline & $\begin{array}{l}\text { T2. Eu me sinto relaxado em } \\
\text { situações sociais não familiares }\end{array}$ & $\begin{array}{l}\text { R2. I feel relaxed in non- } \\
\text { familiar social situations }\end{array}$ & \\
\hline \multirow{2}{*}{$\begin{array}{l}\text { 2. I try to avoid } \\
\text { situations which force } \\
\text { me to be very } \\
\text { sociable }\end{array}$} & $\begin{array}{l}\text { T1. Eu tento evitar situações que } \\
\text { me obriguem a ser muito } \\
\text { sociável }\end{array}$ & $\begin{array}{l}\text { B1. I try to avoid situations in } \\
\text { which I'm supposed to be } \\
\text { sociable }\end{array}$ & $\begin{array}{c}\text { Eu tento evitar situações que } \\
\text { me obriguem a ser muito } \\
\text { sociável }\end{array}$ \\
\hline & $\begin{array}{l}\text { T2. Eu tento evitar situações que } \\
\text { me forcem a ser mais sociável }\end{array}$ & $\begin{array}{l}\text { B2. I tend to avoid situations in } \\
\text { which I am forced to be more } \\
\text { sociable }\end{array}$ & \\
\hline \multirow{2}{*}{$\begin{array}{l}\text { 3. It is easy for me to } \\
\text { relax when I am with } \\
\text { strangers }\end{array}$} & $\begin{array}{l}\text { T1. É fácil relaxar quando estou } \\
\text { com estranhos }\end{array}$ & $\begin{array}{l}\text { B1. It is easy for me to relax } \\
\text { when I'm among strangers }\end{array}$ & $\begin{array}{c}\text { É fácil ficar à vontade quando } \\
\text { estou com estranhos }\end{array}$ \\
\hline & $\begin{array}{l}\text { T2. É fácil relaxar quando estou } \\
\text { com estranhos }\end{array}$ & $\begin{array}{l}\text { R2. It's easy to relax when I'm } \\
\text { in the company of strangers }\end{array}$ & \\
\hline \multirow[t]{2}{*}{$\begin{array}{l}\text { 4. I have no particular } \\
\text { desire to avoid people }\end{array}$} & $\begin{array}{l}\text { T1. Eu não tenho qualquer } \\
\text { desejo de evitar pessoas }\end{array}$ & B1. I don't try to avoid people & $\begin{array}{c}\text { Eu não tenho qualquer desejo } \\
\text { de evitar pessoas }\end{array}$ \\
\hline & $\begin{array}{c}\text { T2. Eu não tenho desejo especial } \\
\text { de evitar pessoas }\end{array}$ & $\begin{array}{l}\text { B2. I have no particular wish to } \\
\text { avoid people }\end{array}$ & \\
\hline \multirow[t]{2}{*}{$\begin{array}{l}\text { 5. I often find social } \\
\text { occasions upsetting }\end{array}$} & $\begin{array}{l}\text { T1. Eu freqüentemente acho } \\
\text { acontecimentos sociais um } \\
\text { transtorno }\end{array}$ & $\begin{array}{l}\text { B1. I frequently find social } \\
\text { occasions stressful }\end{array}$ & $\begin{array}{c}\text { Eu freqüentemente acho } \\
\text { situações sociais uma chateação }\end{array}$ \\
\hline & $\begin{array}{l}\text { T2. Eu freqüentemente } \\
\text { considero os compromissos } \\
\text { sociais desconcertantes }\end{array}$ & $\begin{array}{l}\text { R2. I frequently consider social } \\
\text { appointments disconcerting }\end{array}$ & \\
\hline \multirow[t]{2}{*}{$\begin{array}{l}\text { 6. I usually feel calm } \\
\text { and comfortable at } \\
\text { social occasion }\end{array}$} & $\begin{array}{l}\text { T1. Eu freqüentemente me sinto } \\
\text { calmo em acontecimentos } \\
\text { sociais }\end{array}$ & $\begin{array}{l}\text { B1. I frequently feel calm in } \\
\text { social situations }\end{array}$ & $\begin{array}{c}\text { Eu geralmente me sinto calmo } \\
\text { e confortável em situações } \\
\text { sociais }\end{array}$ \\
\hline & $\begin{array}{l}\text { T2. Eu geralmente me sinto } \\
\text { calmo e confortável em } \\
\text { acontecimentos sociais }\end{array}$ & $\begin{array}{l}\text { B2. I usually feel calm and } \\
\text { comfortable during social } \\
\text { events }\end{array}$ & \\
\hline
\end{tabular}




\begin{tabular}{lcc}
\hline Versão original & \multicolumn{1}{c}{ Traduções } & Retrotraduçóes \\
\hline $\begin{array}{l}\text { 7. I am usually at ease } \\
\text { when talking to } \\
\text { someone of the } \\
\text { opposite sex }\end{array}$ & $\begin{array}{c}\text { T1. Eu geralmente me sinto } \\
\text { tranqüilo quando converso com } \\
\text { alguém do sexo oposto }\end{array}$ & $\begin{array}{c}\text { B1. I generally feel comfortable } \\
\text { when talking to someone of the } \\
\text { opposite sex }\end{array}$ \\
$\begin{array}{l}\text { T2. Eu geralmente fico à } \\
\text { vontade conversando com } \\
\text { alguém do sexo oposto }\end{array}$ & $\begin{array}{c}\text { B2. I am usually comfortable } \\
\text { talking to a member of the } \\
\text { opposite sex }\end{array}$ \\
$\begin{array}{l}\text { T1. I try to avoid } \\
\text { talking to people }\end{array}$ & pessoas que não conheço bem & $\begin{array}{c}\text { B1. I avoid talking to people } \\
\text { which I don't know well }\end{array}$
\end{tabular}
uns unless I know them well

\section{If the chance} comes to meet new people, I often take it

10. I often feel nervous or tense in casual get-togethers in which both sexes are present

\section{I am usually} nervous with people unless I know them well

12. I usually feel relaxed when I am with a group of people

13. I often want to get away from people

14. I usually feel uncomfortable when I am in a group of people I don't know
T2. Eu tento evitar conversar com pessoas, a menos que as conheça bem

T1. Se tenho uma chance de conhecer pessoas, eu geralmente aproveito

T2. Se aparece a oportunidade de conhecer novas pessoas, eu freqüentemente aceito

T1. Eu freqüentemente fico nervoso ou tenso em encontros casuais em que ambos os sexos estão presentes

T2. Eu freqüentemente me sinto nervoso ou tenso em reuniões informais onde pessoas de ambos os sexos estão presentes

T1. Eu geralmente me sinto nervoso com pessoas que não conheço bem

T2. Eu geralmente fico nervoso com pessoas, a menos que as conheça bem

T1. Eu geralmente ficoà vontade quando estou com um grupo de pessoas

T2. Eu geralmente me sinto relaxado quando estou com um grupo de pessoas

T1. Eu freqüentemente quero fugir das pessoas

$\mathrm{T} 2$. Eu freqüentemente quero fugir das pessoas

T1. Eu freqüentemente me sinto desconfortável quando estou em um grupo de pessoas que não conheço

T2. Eu geralmente me sinto desconfortável quando estou em um grupo de pessoas que não conheço
B2. I tend to avoid talking to people, unless I know them well

B1. If I have a chance to meet new people I take it

B2. If the opportunity to meet new people shows up, I usually agree to

B1. I frequently feel nervous or tense In occasional encounters in which both male and female people are present

B2. I frequently feel nervous or tense in informal meetings where people from both sexes are present

B1. I generally feel nervous with people that I don't know well

B2. I am usually nervous around people, unless I know them well

B1. I generally feel comfortable in a group of people

B2. I usually feel relaxed when I'm with a group of people

\section{B1. I frequently want to get} away from people

B2. I frequently want to run away from people

B1. I frequently feel uncomfortable in a group of people who I don't know well

B2. I generally feel uncomfortable when I'm around a group of people I don't know

\section{Versão sintética}

Eu geralmente fíco à vontade conversando com alguém do sexo oposto

Eu tento evitar conversar com pessoas, a menos que as conheça bem

Eu freqüentemente aproveitoa oportunidade de conhecer novas pessoas

$\mathrm{Eu}$ freqüentemente me sinto nervoso ou tenso em reuniões informais onde pessoas de ambos os sexos estão presentes

Eu geralmente fico nervoso com pessoas, a menos que as conheça bem

Eu geralmente fico à vontade quando estou com um grupo de pessoas

Eu freqüentemente quero fugir das pessoas

Eu geralmente me sinto desconfortável quando estou em um grupo de pessoas que não conheço 


\begin{tabular}{lccc}
\hline Versão original & Traduções & Retrotraduções & Versão sintética \\
\hline $\begin{array}{l}\text { 15. I usually feel } \\
\text { relaxed when I meet } \\
\begin{array}{l}\text { someone for the first } \\
\text { time }\end{array}\end{array}$ & $\begin{array}{c}\text { T1. Eu geralmente fico à } \\
\text { vontade quando conheço uma } \\
\text { pessoa pela primeira vez }\end{array}$ & $\begin{array}{c}\text { B1. I generally feel comfortable } \\
\text { the first time I meet someone }\end{array}$ & $\begin{array}{c}\text { Eu geralmente fico à vontade } \\
\text { quando conheço alguém pela } \\
\text { primeira vez }\end{array}$
\end{tabular}

16. Being introduced to people makes me tense and nervous

17. Even though a room is full of strangers, I may enter it anyway

18. I would avoid walking up and joining a large group of people

19. When my superiors want to talk with me, I talk willingly

20. I often feel on the edge when I am with a group of people

21. I tend to withdraw from people

\section{I don't mind} talking to people at parties or social gatherings

23. I am seldom at ease in a large group of people
T2. Eu geralmente me sinto relaxado quando conheço alguém pela primeira vez

T1. Ser apresentado a pessoas me deixa tenso e nervoso

T2. Ser apresentado às pessoas me deixa tenso e nervoso

T1. Mesmo com uma sala cheia de estranhos, eu consigo entrar com facilidade

T2. Mesmo se um lugar estiver cheio de pessoas estranhas, eu consigo entrar

T1. Eu evitaria me dirigir ou me juntar a um grande grupo de pessoas

T2. Eu evitaria caminhar e juntar-me a um grupo grande de pessoas

T1. Quando os meus superiores querem falar comigo, falo de bom grado

T2. Quando meus superiores querem falar comigo, eu falo com gosto

T1. Eu freqüentemente me sinto nervoso quando estou em um grupo de pessoas

T2. Eu freqüentemente me sinto impaciente quando estou com um grupo de pessoas

T1. Eu tenho a tendência a afastar as pessoas

T2. Eu tendo a me afastar das pessoas

T1. Eu não me importo em falar com pessoas em festas ou encontros sociais

T2. Eu não faço questão de conversar com pessoas em festas e reuniões sociais

T1. Eu raramente fico à vontade em um grande grupo de pessoas

T2. Eu raramente estou tranqüilo em um grupo grande de pessoas
B2. I generally feel relaxed when I meet someone for the first time

B1. Being introduced to other people makes me tense and nervous

B2. Being introduced to people makes me tense and nervous

B1. Even in a room full of strangers, I can go in easily

B2. Even if a place is crowded with strangers, I am able to get in

B1. I would avoid to address or join a large group of people

B2. I would avoid taking a walk and joining a group of people

B1. When my superiors want to talk to me, I do it willingly

B2. When my superiors want to talk to me, I am happy to do so

B1. I frequently feel nervous in a group of people

B2. I frequently feel impatient when I'm in a group of people
B1. I tend to stay away from people

B2. I tend to push people away

$$
\begin{aligned}
& \text { B1. I don't mind talking to } \\
& \text { people in parties or social } \\
& \text { occasions }
\end{aligned}
$$

B2. I make no effort to talk to people in parties and social gatherings

B1. I seldom feel comfortable in Eu raramente fico à vontade em a large group of people

B2. I am rarely at ease in a large group of people
Ser apresentado a pessoas me deixa tenso e nervoso

Mesmo com uma sala cheia de desconhecidos, eu consigo entrar com facilidade

Eu evitaria abordar um grande grupo de pessoas e me juntar a elas

Quando os meus superiores querem falar comigo, falo de bom grado

Eu freqüentemente me sinto extremamente nervoso quando estou em um grupo de pessoas

Eu tendo a me afastar das pessoas

Eu não me importo em falar com pessoas em festas ou encontros sociais um grande grupo de pessoas 


\begin{tabular}{|c|c|c|c|}
\hline Versão original & Traduções & Retrotraduções & Versão sintética \\
\hline \multirow{2}{*}{$\begin{array}{l}\text { 24. I often think up } \\
\text { excuses in order to } \\
\text { avoid social } \\
\text { engagements }\end{array}$} & $\begin{array}{c}\text { T1. Eu freqüentemente procuro } \\
\text { desculpas para evitar } \\
\text { compromissos sociais }\end{array}$ & $\begin{array}{l}\text { B1. I frequently look for } \\
\text { excuses to avoid social } \\
\text { occasions }\end{array}$ & $\begin{array}{c}\text { Eu freqüentemente invento } \\
\text { desculpas para evitar } \\
\text { compromissos sociais }\end{array}$ \\
\hline & $\begin{array}{l}\text { T2. Eu freqüentemente invento } \\
\text { desculpas para evitar } \\
\text { compromissos sociais }\end{array}$ & $\begin{array}{l}\text { B2. I frequently make up } \\
\text { excuses to avoid social } \\
\text { appointments }\end{array}$ & \\
\hline \multirow{2}{*}{$\begin{array}{l}\text { 25. I sometimes take } \\
\text { the responsibility for } \\
\text { introducing people to } \\
\text { each other }\end{array}$} & $\begin{array}{l}\text { T1. Às vezes assumo a } \\
\text { responsabilidade de apresentar } \\
\text { pessoas }\end{array}$ & $\begin{array}{l}\text { B1. I sometimes take over the } \\
\text { responsibility of introducing } \\
\text { people }\end{array}$ & $\begin{array}{l}\text { Às vezes eu assumo a } \\
\text { responsabilidade de apresentar } \\
\text { pessoas }\end{array}$ \\
\hline & $\begin{array}{c}\text { T2. De vez em quando eu } \\
\text { assumo a responsabilidade de } \\
\text { apresentar pessoas umas às } \\
\text { outras }\end{array}$ & $\begin{array}{l}\text { B2. Once in a while I assume } \\
\text { the responsibility of introducing } \\
\text { people to each other }\end{array}$ & \\
\hline \multirow{2}{*}{$\begin{array}{l}26 . \text { I try to avoid } \\
\text { formal social } \\
\text { occasions }\end{array}$} & $\begin{array}{l}\text { T1. Eu tento evitar ocasiões } \\
\text { sociais formais }\end{array}$ & $\begin{array}{l}\text { B1. I try to avoid formal social } \\
\text { occasions }\end{array}$ & $\begin{array}{l}\text { Eu tento evitar ocasiões sociais } \\
\text { formais }\end{array}$ \\
\hline & $\begin{array}{l}\text { T2. Eu tento evitar } \\
\text { compromissos sociais formais }\end{array}$ & $\begin{array}{l}\text { B2. I tend to avoid formal social } \\
\text { appointments }\end{array}$ & \\
\hline \multirow[t]{2}{*}{$\begin{array}{l}\text { 27. I usually go to } \\
\text { whatever social } \\
\text { engagements I have }\end{array}$} & $\begin{array}{l}\text { T1. Eu freqüentemente vou a } \\
\text { qualquer compromisso social } \\
\text { que tenho }\end{array}$ & $\begin{array}{l}\text { B1. I frequently go to any social } \\
\text { appointments I have }\end{array}$ & $\begin{array}{c}\text { Eu geralmente vou a qualquer } \\
\text { compromisso social que eu } \\
\text { tenha }\end{array}$ \\
\hline & $\begin{array}{c}\text { T2. Eu geralmente vou a } \\
\text { qualquer compromisso social } \\
\text { que eu tenha }\end{array}$ & $\begin{array}{l}\text { B2. I generally attend any social } \\
\text { appointment I have }\end{array}$ & \\
\hline \multirow{2}{*}{$\begin{array}{l}28 \text {. I find easy to } \\
\text { relax with other } \\
\text { people }\end{array}$} & $\begin{array}{l}\text { T1. Eu acho fácil ficar à vontade } \\
\text { com outras pessoas }\end{array}$ & $\begin{array}{l}\text { B1. I find it easy to feel } \\
\text { comfortable with other people }\end{array}$ & $\begin{array}{l}\text { Eu acho fácil ficar à vontade } \\
\text { com outras pessoas }\end{array}$ \\
\hline & $\begin{array}{l}\text { T2. Eu acho fácil relaxar com } \\
\text { outras pessoas }\end{array}$ & $\begin{array}{l}\text { B2. I find it easy to relax around } \\
\text { other people }\end{array}$ & \\
\hline
\end{tabular}

A versão feita pelos tradutores para os itens 2,3 , $13,14,16,24$ e 27 foram idênticas ou muito semelhantes. Em alguns itens, uma versão teve prioridade sobre outra, ou ambas foram combinadas.

No item 1, "I feel relaxed even in unfamiliar social situations", mantivemos a tradução T2, ao invés da T1, uma vez que o termo "não-familiar" em português brasileiro também significa "uma pessoa que não é parente", e poderia causar alguma confusão no entendimento da frase. O termo "não-familiaridade" em português brasileiro refere-se a "não conhecer alguém muito bem" e pareceu mais adequado quando comparado ao termo original "unfamiliar".

No item 4, embora a tradução T2 de "special desire" para "desejo especial" seja uma tradução literal, o termo T1 "desejo qualquer" parece mais adequado à língua portuguesa.

No item 5, não concordamos com os tradutores em relação à palavra "upsetting", que foi traduzida por "transtorno" e "desconcertante". Durante o desenvolvimento da versão sintética, em nossa opinião, a palavra original referia-se a "algo que incomoda". Desta forma, substituímos por "chateação", que parece ter o mesmo significado de "upsetting".

No item 6, "I usually feel calm and comfortable at social occasion," substituímos as duas traduções do termo "social occasion", uma vez que o termo usado em português, "acontecimentos sociais", parece indicar "um grande evento social". O termo foi substituído por "situações sociais". Além disso, mantivemos a estrutura da tradução T2, uma vez que a T1 não traduziu a palavra "comfortable".

Os enunciados $1,7,12,15,23$ e 28 contêm os termos "relaxed" e "at ease", traduzidos por palavras semelhantes, como "tranqüilo" e "relaxado". Entretanto, optamos pela tradução "à vontade", que significa "sentirse confortável", diferente das primeiras traduções, expressando "sonolento ou mal vestido".

Nos itens 8 e 11, as duas traduções T1 não incluíram a tradução literal da palavra "unless", mas substituíram a palavra por termos semelhantes em português. Consideramos a tradução T2 "a menos que" mais adequada para preservar a estrutura da frase. 
No item 9, optamos pela palavra T1 "aproveito", que tem significado semelhante ao de "take it", ao invés da palavra "aceito". Também optamos por outra estrutura da sentença, já que as opções sugeridas pelos tradutores eram um tanto "pomposas". Elaboramos a frase da seguinte forma: "Eu freqüentemente aproveito a oportunidade de conhecer novas pessoas".

No item 10, optamos pela tradução T2, já que o termo "reuniões informais" parecia mais semelhante à expressão inglesa "casual get together" do que a tradução T1, que optou por "encontros casuais". Em português brasileiro, "casual" é uma palavra com significado semelhante à "informal", e pode não ser completamente entendida.

No item 17, escolhemos a tradução T1 devido à falta de tradução da palavra "easily". Também substituímos as duas traduções da palavra inglesa "strangers" pela palavra "desconhecidos". T1 e T2 utilizaram a palavra "estranhos", que pode denotar "pessoas bizarras".

O item 18 foi particularmente difícil para os tradutores. O termo "walk up" não pôde ser completamente entendido, uma vez que a maioria dos dicionários não contém este verbo. $\mathrm{O}$ verbo é mais freqüentemente traduzido por "caminhar". Fomos orientados por um falante nativo dos EUA para traduzir o verbo por "abordar".

No enunciado 19, a palavra "willingly" teve duas traduções distintas: "com gosto" e "bom grado". As traduções apresentam o mesmo significado, mas a primeira parecia se adequar melhor somente porque é uma palavra mais usual em português brasileiro para denotar alguém que não tem nenhum problema em fazer algo, e por isso o faz com prazer.

O termo "on the edge" no item 20 também apresentou duas traduções: "nervosa" e "impaciente". Para a versão sintética, os autores criaram outra expressão, "extremamente nervosa", que dá a impressão de uma pessoa que fica verdadeiramente incomodada pela situação, com a sensação de estar em seu limite, e considerou-se que esta teria um significado mais próximo do termo original.

No item 21, a sentença "I tend to withdraw from people" foi melhor traduzida na tradução T2, "eu tendo a me afastar da pessoas," do que na T1, "eu tenho a tendência a afastar as pessoas". Na primeira tradução, o indivíduo voluntariamente se isola das pessoas, é ativo no processo de isolamento; na segunda, o indivíduo é isolado independente de sua vontade.

No enunciado 22, a expressão "I don't mind" tem duas traduções para o português brasileiro: "eu não me importo" (T1) e "eu não faço questão" (T2). A primeira tradução indica que "eu não tenho problema em fazer algo", e a segunda, que "fazer algo não é importante para mim". A primeira tradução pareceu mais próxima do significado em língua inglesa, que parece se referir a "uma falta de problema em fazer algo".

No item 25, "introducing people to each other" foi traduzido por T1 como "apresentar pessoas" e, de acordo com T2, como "apresentar pessoas umas às outras". As duas têm o mesmo significado de "apresentar pessoas"; no entanto, a segunda tradução não foi considerada gramaticalmente correta porque, em português brasileiro, representa uma sentença redundante.

No item 26, a expressão "social occasions" foi traduzida por "ocasiões formais" (T1) e "compromissos formais" (T2). A primeira refere-se a "encontros formais", e a segunda, a "compromissos formais". Considerou-se que a T1 apresentava significado mais semelhante a "ocasiões sociais".

Durante o pré-teste com os participantes, nosso objetivo foi modificar qualquer termo ou sentença que pudesse ser de difícil compreensão. A maioria das sugestões derivou do grupo com ensino superior em relação à similaridade dos enunciados, que pareceram um tanto repetitivos. Esta alegação não se observou nos outros grupos. Houve somente uma pessoa no grupo de estudo básico que apresentou alguma dificuldade de compreensão do significado de "ocasiões sociais". Como este caso representou uma exceção no grupo de pessoas com o mesmo nível de escolaridade, optamos por não incluir uma explicação do significado desta expressão na escala. As pessoas com nível universitário pareceram ser os participantes mais exigentes no estudo, e isso pode ser considerado um viés, uma vez que eles naturalmente se sentiram mais propensos a prestar atenção a qualquer possível problema com os itens do que as pessoas com nível mais baixo de escolaridade, que geralmente prestam mais atenção ao sentido geral das sentenças.

\section{Discussão}

Ainda é difícil apontar a melhor metodologia para realizar equivalência semântica. Nosso trabalho destacou este processo utilizando duas traduções independentes e duas retrotraduções. Com base nas diretrizes propostas na literatura, enfatizamos a equivalência semântica, ao invés da equivalência literal dos termos para expressar conceitos da nova população ${ }^{29}$.

Diferenças em definições, crenças e comportamentos em relação a muitas populações exigem que os instrumentos desenvolvidos para outros contextos culturais sejam precedidos por avaliação meticulosa da equivalência entre o original e sua versão adaptada $^{34}$. O uso de um instrumento para outra cultura é uma tentativa de investigar um sintoma em uma cultura específica, porém pode por vezes ser questionado, uma 
vez que nunca removerá todos os vieses quando adaptado para uso em outra população ${ }^{35}$.

Em nosso estudo, tivemos que discutir algumas questões relativas a termos específicos da língua inglesa, como "on the edge" e "to walk up", que não foram facilmente traduzidos para o português brasileiro. Portanto, foram transformados em termos locais de acordo com nossa cultura. Também verificamos que alguns tradutores tendem a excluir algumas palavras que no português brasileiro corrente podem ser omitidas; no entanto, optamos pela manutenção da estrutura do instrumento original com o mínimo possível de alterações.

Alguns problemas geralmente encontrados na tradução de instrumentos do idioma original para a línguaalvo resultam do desconhecimento por parte dos tradutores em relação à área de pesquisa. A diferença em termos de significado subjetivo de palavras e termos que existem em inglês para outra língua e cultura pode não fazer sentido em uma tradução direta, retendo muitas das formas literalmente gramaticais da língua-fonte ${ }^{13,36}$. Problemas como esse têm sido uma causa potencial de contaminação de dados em muitos estudos transculturais, além de serem um obstáculo contra a equivalência ${ }^{15}$.

Observamos que o uso de dois tradutores foi extremamente útil na construção da versão sintética, uma vez que as versões puderam ser confrontadas e discutidas. Quando discrepâncias foram identificadas, discutimos extensamente as razões e propusemos soluções. Weeks et al..$^{15}$ orientam os autores a repetir o procedimento por diversas etapas, já que os tradutores trabalham com os esforços de seus predecessores. Uma limitação de nosso estudo foi o pequeno número de indivíduos $(\mathrm{n}=24)$ que leram e avaliaram o instrumento. Esta limitação foi atenuada pelo uso de pessoas de diferentes níveis de escolaridade que desconheciam qualquer versão da SADS.

Apesar de termos recrutado avaliadores independentes e "cegos", seria interessante pedir a um especialista que auxilie os autores no processo de equivalência semântica. $\mathrm{O}$ especialista poderia lançar luz sobre questões que não pudemos identificar, uma vez que é raro que os autores sejam inteiramente cegos ao estudo. Esta pode ser considerada outra limitação de nosso estudo.

A aplicação do pré-teste mostrou que os participantes entenderam as situações descritas na escala, e muitas vezes se identificaram como tendo ações ou sentimentos semelhantes aos dos enunciados em relação à evitação e mal-estar social. É possível que este instrumento, ao invés de diagnosticar fobia social, identifique dificuldades sociais comuns apresentadas por um grande grupo de pessoas.

A adaptação transcultural e a equivalência semântica são apenas a primeira etapa na validação de um instrumento em qualquer idioma. Para um uso adequado do instrumento, este deve ter boa confiabilidade e validade, além de boa sensibilidade e especificação ${ }^{24}$. Este estudo é o primeiro passo rumo à determinação desses fatores com a aplicação da versão em português brasileiro da SADS em uma grande amostra da população brasileira.

\section{Referências}

1. American Psychiatric Association (APA). DSM IV-TR Manual diagnóstico e estatístico de transtornos mentais: DSM IV-TR. Porto Alegre: Artmed; 2003

2. Marks IM, Gelder MG. Different ages of onset in varieties of phobia. Am J Psychiatry. 1966;123:218-21.

3. Heckelman LR, Schneier FR. Diagnostic issues. In: Heimberg RG, Liebowitz MR, Hope DA, Schneier FR, editors. Social phobia: diagnosis, assessment and treatment. New York: Guilford; 1995. p. 165-85.

4. Bobes J, Badía X, Luque A, Garcia M, Paz Gonzalez M, Dal-Re R. Validación de las versiones en español de los cuestionarios Liebowitz social anxiety scale, Social Anxiety and Distress Scale y Sheehan Disability Inventory para la evaluación de la fobia social. Med Clin (Barc). 1999;112:530-8.

5. Yao SN, Note I, Fanget F, Albuisson E, Bouvard M, Jalenques I, et al. Social anxiety in patients with social phobia: validation of the Liebowitz social anxiety scale: the French version. Encephale. 1999;25:429-35.

6. Soykan C, Ozgüven HD, Gençöz T. 2003. Liebowitz Social Anxiety Scale: the Turkish Version. Psychol Rep. 2003;93:1059-69.

7. Terra MB, Barros HM, Stein AT, Figueira I, Athayde LD, Gonçalves Mde S, et al. Internal consistency and factor structure of the portuguese version of the Liebowitz Social Anxiety Scale among alcoholic patients. Rev Bras Psiquiatr. 2006;28:265-9.

8. Mas Pons R, Escriba Agüir V. La versión castellana de la escala The Nursing Stress Scale. Proceso de adaptación transcultural. Rev Esp Salud Publica. 1998;72:529-38.

9. Yu DS, Lee DT, Woo J. Issues and challenges of instrument translation. West J Nurs Res. 2004;26:307-20.

10. Fletcher RH, Fletcher SW, Wagner EH. Epidemiologia clínica. Barcelona: Consulta; 1989.

11. Lima LA, Gonçalves S, Lovisi G, Pereira BB. Validação transcultural da Escala de Avaliação de Limitações do Comportamento Social - SBS-BR. Rev Psiq Clin. 2003;30:126-38.

12. Picon P. A publicação de estudos brasileiros de validação de medidas de aferição em psiquiatria. Rev Psiq RS. 2006;28:117-9.

13. Brislin RW. Back-translation for cross-cultural research. J Cross Cult Psychol. 1970;1(3):185-216.

14. Werner O, Campbell D. Translating, working though interpreters and the problem of decentering. In: Naroll R, Cohen R, editors. A handbook of method in cultural anthropology. New York: American Museum of Natural History; 1970. p. 398-420.

15. Weeks A, Swerissen H, Belfrage J. Issues, challenges, and solutions in translating study instruments. Eval Rev. 2007;31;153-65.

16. Watson D, Friend R. Measurement of social-evaluative anxiety. J Consult Clin Psychol. 1969;33:448-57.

17. Orsillo SM. Measures for social phobia. In: Antony MM, Orsillo SM, Roemer L, editors. Practitioner's guide to empirically based measures of anxiety. New York: Kluwer; 2001. p. 165-85.

18. Turner SM, McCanna M, Beidel DC. Validity of the social avoidance and distress and the fear of negative evaluation scales. Behav Res Ther. 1987;25:113-5.

19. Gauer GJ, Picon P, Vasconcellos SJ, Turner SM, Beidel DC. Validation of the social phobia and anxiety inventory for children (SPAI-C) in a sample of Brazilian children. Braz J Med Biol Res. 2005;38:795-800.

20. Versiani M, Nardi AE, Mundim FD, Pinto S, Saboya E, Kovacs R. The long-term treatment of social phobia with moclobemide. Int Clin Psychopharmacol. 1966;11:Suppl 3:83-8. 
21. Versiani M, Mundim FD, Nardi AE, Liebowitz M. Tranylcypromine in social phobia. J Clin Psychopharmacol. 1988;8:279-83.

22. Versiani M, Nardi AE, Mundim FD, Alves AB, Liebowitz M, Amrein R. Pharmacotherapy of social phobia. Br J Psychiatry. 1992;161:353-60.

23. Nardi AE. Social anxiety disorder has social and economic burden. BMJ. 2003;29:327:1287.

24. Berger W, Mendlowicz MV, Souza WF, Figueira I. Equivalência semântica da versão em português da Post-Traumatic Stress Disorder Checklist-Civilian Version (PCL-C) para rastreamento do transtorno de estresse pós-traumático. Rev Psiquiatr RS. 2004;26:167-5.

25. Picon P, Gauer GJC, Fachel JMG, Manfro GG. Desenvolvimento da versão em portugues do Social Phobia and Anxiety Inventory (SPAI). Rev Psiquiat RS. 2005;27:40-50.

26. Menezes GB, Fontenelle LF, Versiani M. Early-onset social anxiety disorder in adults: clinical and therapeutic features. Rev Bras Psiquiatr. 2005;27:32-6.

27. Nardi AE. Early diagnosis can decrease the social and economic burden of social anxiety disorder. Aust $\mathrm{N}$ Z J Psychiatry. 2005;39:641-2.

28. Valença AM, Nardi AE, Nascimento I, Lopes FL, Freire RC, Mezzasalma MA, et al. Do social anxiety disorder patients belong to a bipolar spectrum subgroup? J Affect Disord. 2005;86:11-8.
29. Vilete L, Figueira I, Coutinho E. Adaptação transcultural para o português do Social Phobia Inventory (SPIN) para utilização entre estudantes adolescentes. Rev Psiquiatr RS. 2006;28:40-8.

30. de Lima Osorio F, Crippa JA, Loureiro SR. A study of the discriminative validity of a screening tool (MINI-SPIN) for social anxiety disorder applied to Brazilian university students. Eur Psychiatry. 2007;22:239-43.

31. Herdman M, Fox-Rushby J, Badia X. A model of equivalence in the cultural adaptation of HRQoL instruments: the universalist approach. Qual Life Res. 1998;7:323-35.

32. Reichenheim ME, Moraes CL, Hasselmann MH. Semantic equivalence of the Portuguese version of the Abuse Assessment Screen tool used for the screening of violence against pregnant women. Rev Saude Publica. 2000;34:610-6.

33. First MB, Spitzer RL, Gibbon M, Williams JBW. Structured clinical interview for DSM-IV axis I disorders: patient edition (SCID I/P, Version 2.0). Washington: APA; 1996.

34. Reichenheim ME, Moraes CL. [Operationalizing the cross-cultural adaptation of epidemiological measurement instruments]. Rev Saude Publica. 2007;41:665-73.

35. Smit J, van den Berg C, Bekker LG, Seedat S, Stein Dj. Translation and cross-cultural adaptation of a mental health battery in an African setting. Afr Health Sci. 2006;6:215-22.

36. Guillemin F. Cross-cultural adaptation and validation of health status measures. Scand J Rheumatol. 1995;24:61-3. 


\section{Anexo}

\section{Escala de esquiva e desconforto social}

Por favor, responda se as seguintes afirmações são verdadeiras ou falsas para você. Circule V para verdadeiro e F para falso.

1. Eu me sinto à vontade em situações sociais que não tenho familiaridade

V F

2. Eu tento evitar situações que me obriguem a ser muito sociável

V F

3. É fácil ficar à vontade quando estou com estranhos

V F

4. Eu não tenho desejo especial de evitar pessoas

V F

5. Eu freqüentemente acho situações sociais uma chateação

V F

6. Eu geralmente me sinto calmo e confortável em situações sociais

V F

7. Eu geralmente fico à vontade conversando com alguém do sexo oposto

8. Eu tento evitar conversar com pessoas, a menos que as conheça bem

9. Eu freqüentemente aproveito a oportunidade de conhecer novas pessoas

10. Eu freqüentemente me sinto nervoso ou tenso em reuniões informais onde pessoas de ambos os sexos estão presentes

11. Eu geralmente fico nervoso com pessoas, a menos que as conheça bem

12. Eu geralmente fico à vontade quando estou com um grupo de pessoas

13. Eu freqüentemente quero fugir das pessoas

14. Eu geralmente me sinto desconfortável quando estou em um grupo de pessoas que não conheço

15. Eu geralmente me sinto à vontade quando conheço alguém pela primeira vez

16. Ser apresentado a pessoas me deixa tenso e nervoso

17. Mesmo com uma sala cheia de desconhecidos, eu consigo entrar com facilidade

18. Eu evitaria abordar um grande grupo de pessoas e me juntar a elas

19. Quando os meus superiores querem falar comigo, falo de bom grado

20. Eu freqüentemente me sinto extremamente nervoso quando estou em um grupo de pessoas

21. Eu tendo a me afastar das pessoas

22. Eu não me importo em falar com pessoas em festas ou encontros sociais

23. Eu raramente fico à vontade em um grande grupo de pessoas

24. Eu freqüentemente invento desculpas para evitar compromissos sociais

25. Às vezes assumo a responsabilidade de apresentar pessoas

26. Eu tento evitar ocasiões sociais formais

27. Eu geralmente vou a qualquer compromisso social que eu tenha

28. Eu acho fácil ficar à vontade com outras pessoas 